\title{
Editorial
}

\section{Cardiac Troponins in Chronic Kidney Disease: A Marker of Global Cardiovascular Risk}

\author{
Muthiah Vaduganathan Deepak L. Bhatt \\ Brigham and Women's Hospital Heart and Vascular Center and Harvard Medical School, Boston, MA, USA
}

Approximately $15 \%$ of people in the US live with chronic kidney disease (CKD) and 500,000 patients require chronic hemodialysis [1]. Despite encouraging recent trends of steady declines in overall mortality in Medicare patients with CKD, cardiovascular (CV) disease continues to account for more than half of the deaths in patients with end-stage renal disease (ESRD) [1], and as such, optimization of $\mathrm{CV}$ outcomes in this population remains a major treatment goal.

Cardiac troponin measurement plays important roles in the early identification, risk stratification, prognostication, management, and monitoring of acute myocardial infarction (MI) in the overall population. However, their frequent elevation in patients with $\mathrm{CKD}$, coupled with the recent advent of newer-generation and increasingly sensitive troponin assays, has made timely and accurate diagnosis of MI in this population challenging. Since elevated troponins are commonly encountered in patients with CKD, we highlight important considerations regarding their practical application in contemporary practice in this high-risk population.

\section{Cardiac Troponins in CKD: Upscaling Our Understanding}

Cardiac troponins may be persistently or "chronically" elevated in patients with CKD, even in those free of overt CV symptoms [2]. The measurement of cardiac troponins

\section{KARGER}

(๑) 2017 S. Karger AG, Basel

E-Mail karger@karger.com

www.karger.com/ajn above the detection limit signifying cardiac myonecrosis may be related to sources other than acute MI and may be unexplained by decreased biomarker clearance alone. Although the pathogenesis of troponin release from myocytes has yet to be fully elucidated, potential contributing mechanisms include pre-existing CV disease (e.g., stable epicardial coronary artery disease, heart failure, left ventricular hypertrophy), subendocardial ischemia, direct myocardial toxicity from uremia (and its associated toxins), and hemodialysis-related stressors [2]. These intervening processes limit the specificity and diagnostic utility of static measurements of troponin in patients with $\mathrm{CKD}$ with acute MI. Clear guidance regarding the optimal management approach to elevated troponins in CKD is lacking.

In 2012, the universal definition of MI put forth by the Third Global MI Task Force represented a concerted effort to provide a structured classification scheme and standardize the use and application of cardiac troponins in a broad range of clinical scenarios [3]. Importantly, the multidisciplinary consensus committee emphasized the need for adjunctive evidence of myocardial ischemia and assessment of the trajectory of this biomarker to further hone the diagnosis of MI. In this issue of the American Journal of Nephrology, Vasudevan et al. [4] carefully apply this suggested approach to a sample of 430 patients presenting with suspected MI who were enrolled in the prospective, multi-center Performance of Triage Cardiac Markers in the Clinical Setting (PEARL) study. This 
observational, "real-world" experience included adult patients presenting to emergency departments with chest pain syndromes. Acute MI was specifically adjudicated and was found to be present in $\sim 20 \%$ of patients by a dedicated team after review of case report forms, electrocardiograms, and interpretation of laboratory data using the universal definition of MI [3]. Given inclusion of data from multiple centers and use of 6 different troponin assays, "scaling" of troponin measurements was employed to standardize results reporting [4]. Scaling refers to the determination of the number of multiples of the assayspecific 99 th percentile upper reference limit above which the patient's measured value lies.

The investigators found that nearly half of patients with CKD had at least one troponin value above the 99th percentile upper reference limit. However, despite this, the rates of adjudicated MI were similar in patients with and without baseline $\mathrm{CKD}$, and these clinical events were characterized by higher peak scaled troponin measurements and a distinct rise and fall in the biomarker values. Vasudevan et al. [4] greatly advance our understanding of cardiac troponins, biomarker trajectories, and assay standardization in the identification of MI in patients with CKD.

As acknowledged by the authors, their findings should be carefully interpreted within the scope of certain limitations. The study focuses on 87 patients with estimated glomerular filtration rates less than $60 \mathrm{~mL} / \mathrm{min} / 1.73 \mathrm{~m}^{2}$, limiting the ability to dissect the influence of CKD stage or hemodialysis status on cardiac troponin test performance and rates of MI. Similarly, a fifth of patients were adjudicated as experiencing an MI, but further classification of type or extent of MI was lacking. Data regarding therapeutic approach and utilization of medical and/or invasive strategies after MI diagnoses may help to clarify real-world practices in this cohort. Finally, the use of troponin data in the adjudication process may have confounded the accurate assessment of MI, potentially artificially improving its test characteristics.

\section{Cardiac Troponins in CKD: Finding Their Place}

The number of commercially available, newer generation troponin assays with greater sensitivities, more rapid detection capabilities, and varying cutoffs and reference ranges are expected to continue to increase in clinical practice. These assays are slowly becoming incorporated into diagnostic pathways and garnering regulatory approval for various indications directly relevant to the management of CKD. In 2004, the US Food and Drug Administration approved the use of a troponin $\mathrm{T}$ assay in risk prediction in patients with ESRD undergoing hemodialysis. In 2017, one of the first high-sensitivity troponin assays has now been cleared for diagnostic use in patients presenting with suspected MI in the US. Paralleling the expanding availability and indications of use of these troponin assays, clinicians will need to become familiar with their optimal integration into management approaches.

"Scaling", as applied by Vasudevan et al. [4], may help facilitate standardization of reporting and interpretation across assays. However, a more nuanced approach to diagnostics in cases of suspected $\mathrm{MI}$ is required beyond single time-point thresholds, especially in challenging populations such as CKD. The overall kinetics of troponin rise/fall and peak biomarker levels may be more useful in the CKD population. Consistent with the universal definition of MI [3], sound clinical reasoning and incorporation of adjunctive evidence of myocardial ischemia are mandatory to appropriately contextualize these laboratory findings.

As the application of troponins in the initial diagnosis of MI in patients with CKD becomes refined, it will continue play a more concrete role in identifying at-risk patients for adverse CV clinical course. Based on data from recent systematic reviews and meta-analyses, elevated levels of troponin I and troponin T were associated with high short-term rates of CV events and mortality in patients with $\mathrm{CKD}$, irrespective of the presence or absence of symptoms or background clinical suspicion of MI $[5,6]$.

\section{Optimizing Cardiovascular Care of Patients with CKD}

Patients with CKD experience high rates of comorbid CV disease, continue to die at a higher rate than non-CKD populations, and contribute disproportionately to healthcare utilization and spending [1]. Despite favorable recent temporal trends in guideline-directed medical therapies [7] and invasive diagnostic and interventional approaches [8] in patients with established CV disease and $\mathrm{CKD}$, these patients continue to face sub-optimal CV outcomes. Since fatal MIs appear to account for only $\sim 6 \%$ of cause-specific mortality in the CKD population based on the US Renal Data System 2015 Annual Data Report [1], a more global CV risk reduction approach may be favored over focus on diagnosis and prevention of MI in isolation. These strategies need to be better defined, but may include aggressive lifestyle modi- 
fication (e.g., smoking cessation, weight reduction), risk factor control (e.g., optimal diabetes and hypertension management), other preventative approaches (e.g., influenza and pneumococcal vaccination, as appropriate), and heightened patient and physician awareness of the signs and symptoms of CV disease. Mapping the natural history, development of safe and efficacious therapies, and amelioration of high $\mathrm{CV}$ events in at-risk patients with CKD identified by elevated cardiac troponins represent unmet clinical needs. A more granular understanding of the pathogenesis of cardiac troponin release in patients with CKD may inform future efforts to improve upon current CV management approaches.

\section{Acknowledgments}

None.

\section{Disclosure Statement}

Dr. Muthiah Vaduganathan has no relevant disclosures related to this work. Dr. Deepak L. Bhatt discloses the following relationships - Advisory Board: Cardax, Elsevier Practice Update Cardiol- ogy, Medscape Cardiology, Regado Biosciences; Board of Directors: Boston VA Research Institute, Society of Cardiovascular Patient Care; Chair: American Heart Association Quality Oversight Committee; Data Monitoring Committees: Duke Clinical Research Institute, Harvard Clinical Research Institute, Mayo Clinic, Population Health Research Institute; Honoraria: American College of Cardiology (Senior Associate Editor, Clinical Trials and News, ACC.org), Belvoir Publications (Editor in Chief, Harvard Heart Letter), Duke Clinical Research Institute (clinical trial steering committees), Harvard Clinical Research Institute (clinical trial steering committee), HMP Communications (Editor in Chief, Journal of Invasive Cardiology), Journal of the American College of Cardiology (Guest Editor; Associate Editor), Population Health Research Institute (clinical trial steering committee), Slack Publications (Chief Medical Editor, Cardiology Today's Intervention), Society of Cardiovascular Patient Care (Secretary/Treasurer), WebMD (CME steering committees); Other: Clinical Cardiology (Deputy Editor), NCDR-ACTION Registry Steering Committee (Chair), VA CART Research and Publications Committee (Chair); Research Funding: Amarin, Amgen, AstraZeneca, Bristol-Myers Squibb, Eisai, Ethicon, Forest Laboratories, Ischemix, Lilly, Medtronic, Pfizer, Roche, Sanofi Aventis, The Medicines Company (including for his role as Co-Chair of the CHAMPION trials); Royalties: Elsevier (Editor, Cardiovascular Intervention: A Companion to Braunwald's Heart Disease); Site Co-Investigator: Biotronik, Boston Scientific, St. Jude Medical; Trustee: American College of Cardiology; Unfunded Research: FlowCo, PLx Pharma, Takeda.

\section{References}

1 Saran R, Li Y, Robinson B, Abbott KC, Agodoa LY, Ayanian J, Bragg-Gresham J, Balkrishnan R, Chen JL, Cope E, Eggers PW, Gillen D, Gipson D, Hailpern SM, Hall YN, He K, Herman W, Heung M, Hirth RA, Hutton D, Jacobsen SJ, Kalantar-Zadeh K, Kovesdy CP, Lu Y, Molnar MZ, Morgenstern H, Nallamothu B, Nguyen DV, O'Hare AM, Plattner B, Pisoni R, Port FK, Rao P, Rhee CM, Sakhuja A, Schaubel DE, Selewski DT, Shahinian V, Sim JJ, Song P, Streja E, Kurella Tamura M, Tentori F, White S, Woodside K, Hirth RA: US renal data system 2015 annual data report: epidemiology of kidney disease in the United States. Am J Kidney Dis 2016;67(3 suppl 1):Svii, S1S305.

2 Vaduganathan M, Bhatt DL: Elevated troponin levels in stable patients undergoing hemodialysis: a red flag or a red herring? Am J Nephrol 2016;43:170-172.
3 Thygesen K, Alpert JS, Jaffe AS, Simoons ML, Chaitman BR, White HD; Joint ESC/ACCF/ AHA/WHF Task Force for Universal Definition of Myocardial Infarction: Third universal definition of myocardial infarction. J Am Coll Cardiol 2012;60:1581-1598.

4 Vasudevan A, Singer AJ, DeFilippi C, Headden G, Schussler JM, Daniels LB, Reed B, Than MP, Birkhahn R, Smith SW, Barrett TW, Arnold W, Peacock WF, McCullough PA: Renal function and scaled troponin in patients presenting to the emergency department with symptoms of myocardial infarction. Am J Nephrol 2017;45:304-309.

5 Michos ED, Wilson LM, Yeh HC, Berger Z, Suarez-Cuervo C, Stacy SR, Bass EB: Prognostic value of cardiac troponin in patients with chronic kidney disease without suspected acute coronary syndrome: a systematic review and meta-analysis. Ann Intern Med 2014;161: 491-501.
6 Stacy SR, Suarez-Cuervo C, Berger Z, Wilson LM, Yeh HC, Bass EB, Michos ED: Role of troponin in patients with chronic kidney disease and suspected acute coronary syndrome: a systematic review. Ann Intern Med 2014; 161:502-512.

7 Pandey A, Golwala H, DeVore AD, Lu D, Madden G, Bhatt DL, Schulte PJ, Heidenreich PA, Yancy CW, Hernandez AF, Fonarow GC: Trends in the use of guideline-directed therapies among dialysis patients hospitalized with systolic heart failure: findings from the American heart association get with the guidelines-heart failure program. JACC Heart Fail 2016;4:649-661.

8 Gupta T, Kolte D, Khera S, Goel K, Aronow WS, Cooper HA, Jain D, Rihal CS, Fonarow GC, Panza JA, Bhatt DL: Management and outcomes of ST-segment elevation myocardial infarction in US renal transplant recipients. JAMA Cardiol 2017, in press. 